\title{
Consultant images
}

John L. Cox

At present there is a somewhat damaging 'splitting' occurring within the NHS 'reforms' - assisted by the media. Consultant psychiatrists, who are regarded as intrusive and paternalistic, administer treatments (mostly medication and ECT) to patients who are not consulted, and are generally thought to know what is best for themselves. General management, which overtly sets out to "change the culture', appears to sustain this irrational harmful and pseudo confrontation.

The NHS reforms, driven primarily by economic and political necessity, have used this apparent abuse of a physician's authority to emphasise the rights of patients/users/ clients for personal autonomy; their need to be actively involved in medical decisions and treatment options whether this relates to cancer surgery, psychotherapy, or restraint from active suicidal activity.

What can be done to halt an apparent decline of health workers' morale and hence standards of patient care? How can the crucial roles of consultant psychiatrists be safeguarded and acknowledged? How can we change the image?

Two ideas have occurred to me.

Within this vexed debate about the leadership role of a consultant in a multidisciplinary team, is not the image of the consultant as 'conductor' a useful metaphor: a 'group' conductor in a psychotherapy context but also an orchestral conductor at a concert hall? After all, not only does 'every group need a leader', but every orchestra requires a conductor. The conductor and the various sections of the orchestra (wind, brass, violins, double bass and percussion) need to play from the same score. The responsibility for the performance, although shared and delegated, is that of the conductor; the conductor's task being to interpret the musical mind of the composer within time and space and so to enhance the aesthetic experience of an audience. An orchestra cannot perform if the woodwind section will not co-operate or if the cellos do not listen to the second violins - or if the conductor has never learnt the score, or falls off the rostrum.

This image of a consultant as conductor, who is not leader of the first violins, may possibly be useful when considering structures necessary for effective clinical work. A consultant-led multi-professional team is necessary if 'at risk' patients are to be identified and optimally managed. Now at last recognised by the NHSE, this component of health care delivery is indeed almost always acknowledged at the clinical 'coal-face' of patient care-certainly at the diagnosis/ assessment phase, which usually determines the resource implications.

The second image which could facilitate the thinking of the working party on consultant responsibility was culled from a paper read at a Philosophy Special Interest Group meeting in Newcastle upon Tyne. A paper by Bryan Vernon, Anglican priest and Chairman of a Mental Health Trust, entitled 'Interconnectedness without Paternalism - a New Jerusalem?' caught my attention.

The abstract read as follows:

"The concept of autonomy has been a successful contender against paternalism, but is in danger of becoming too powerful. Without due attention to the demands of society, unfettered individualism becomes self-destructive. This notes the pressure on autonomy from cost-containment sources and puts forward a richer understanding of the self as other-regarding"

Quite so - but is it 'either/or'? The search for autonomy is a particularly Western preoccupation (compare Japan); is not the image of 'medical parentalism', or even 'maternalism' more useful as an accurate account of best practice? Space creating, enabling, sustaining and even 'letting go', is perhaps closer to good doctoring and to the role of a consultant than intrusive aggressive paternalism. Parentalism and maternalism implies a balance between an appropriate autocratic use of professionalism, (the wish of the patient to be healed by a knowledgeable healer) and the need to preserve the essential individuality and autonomy of a patient. 
These two images arise from a certain familiarity with musical performance as well as with leadership issues in a day hospital or an admission ward. They cannot of course be taken too far. Indeed several other ideas might also stimulate debate. An image of the doctor as 'manager', or manager as 'consultant', might also be usefully explored.

There is, however, a need for the College, and government, to acknowledge good practice and recognise that a consultant-based team is in most instances also optimally consultant-led. Most other mental health professionals do not seek, nor do they wish for, such responsibility - nor are they paid for it. This 'parental' leadership, embedded in NHS tradition, is unlikely to be radically changed by a cultural shift; it should however be earned and sustained through specific continuing education, and in particular by training within a clinical setting.

It is hoped that the President's working party will continue to address these issues and that its report will be heeded by managers and managed.

John L. Cox, Dean, Royal College of Psychiatrists

\title{
Sexual harassment of staff by patients in mental health units
}

\author{
Maria B. Tomé de la Granja
}

The Health and Safety Executive (1992) defines violence against staff as "any incident in which an employee is threatened or assaulted by a member of the public in circumstances arising out of the course of his or her employment". Verbal abuse and threats are, as the Health and Safety Executive notes, the most common types of incidents, and staff have the common-law right to be protected from such incidents in the course of their work. While the literature on physical violence against mental health professionals is quite large and expanding (see, for example, Health and Safety Commission, 1987; Shepherd, 1994; Wykes, 1994), comparatively little emphasis has been placed on verbal assaults, although these may be extremely distressing.

A common problem in our unit (a rehabilitation unit associated with a community sectorised service in an inner city) is sexually provocative comments to female and, rarely, male staff by patients. An informal poll of colleagues in all disciplines indicates that our unit is far from unique in this. A frequent feeling in the victim of such comments, which amount to sexual harassment, is of frustration because one does not know how to deal with it, and particularly because one is unsure that the matter will be taken seriously. We are implementing a unit policy on sexual harassment by patients. The policy is given below, as 'Eight Commandments'. It seeks to complement and not replace existing policies on sexual harassment at work.

(1) We regard it as fundamental that members of staff should be able to perform their duties and patients their treatment and rehabilitation without sexual harassment and that the unit will not tolerate such behaviour. In other words, sexual harassment is not to be regarded merely as an occupational hazard. It is important that this message is made clear to all, including patients.

(2) Sexual harassment occurs when a member of staff suffers annoyance and/ or impedance in his or her work due to words or actions that make it plain that the member of staff is considered not as 